\title{
Unreviewed Reports
}

\begin{abstract}
Atypical polyneuropathy complicating rectal carcinoma
A 60 year old man presented with a mixed asymmetrical peripheral neuropathy and bilateral lower motor neuron facial palsy. Cerebrospinal fluid analysis and electroneurographic studies suggested a carcinomatous neuropathy. A large rectal carcinoma (Duke's stage C) was partially excised at laparotomy. The neuropathy progressed gradually, and he died 14 weeks postoperatively. At necropsy tumour was confined to the pelvis. Although a mixed peripheral neuropathy is the most common non-metastatic complication of cancer, we have found only two other reports of an association with rectal tumour and only one of the facial nerve being affected by any tumour.-D C WHEELER, A C B wICKS, Leicester General Hospital, Leicester LE5 4PW. (Accepted 24 September 1984)
\end{abstract}

1 Croft $\mathrm{PB}$, Wilkinson $M$. The incidence of carcinomatous neuromyopathy in patients with various types of carcinoma. Brain 1965;88:427-34.

\section{Reaction to oral acyclovir}

A 29 year old woman was treated for primary genital herpes simplex with oral acyclovir $200 \mathrm{mg}$ five times daily for five days. Three days later she developed pruritic facial erythema, which rapidly progressed in a caudal direction, evolving into a maculopapular eruption with multiple foci of micropustules. The eruption subsided over two weeks, with topical and systemic steroids. Skin biopsy findings were consistent with a drug reaction without evidence of erythema multiforme. Although a patch test to acyclovir cream yielded negative results, the close temporal relation together with the clinical and histopathological features suggested that oral acyclovir was responsible. - C E H GRATTAN, J BOYLE, Department of Dermatology, Bristol Royal Infirmary, Bristol BS2 8HW. (Accepted 9 October 1984)

\section{Thalidomide and multiple cerebral aneurysms: fortuitous or} causal association?

A 20 year old woman, married with healthy 5 month old twins, was referred to this unit with subarachnoid haemorrhage. She was a known thalidomide victim with long phocomelia and polydactyly as the only detectable stigmata of thalidomide embryopathy. Diagnosis of subarachnoid haemorrhage was confirmed by lumbar puncture and computed tomography. Cerebral angiography showed at least three aneurysms.' One week post ictus an anterior communicating artery aneurysm, undoubtedly the source of the bleed, was clipped. She made an uneventful recovery. Check angiography with a view to elective surgery was arranged for three months later.-E M HASSAN, H H GOSSMAN, Neurosurgical Unit, Freedom Fields Hospital, Plymouth PL4 7JJ. (Accepted 23 October 1984)

1 Smithells RW. Defects and disabilities of thalidomide children. Br Med f 1973;i:269.

\section{Primary familial dilated cardiomyopathy}

Two brothers, separated for many years, presented in their 30s with congestive cardiac failure. Both had dilated cardiomyopathy of unknown cause, confirmed on echocardiography; there was no other relevant family history. One failed to respond to medical treatment and at necropsy was found to have biventricular dilatation with little hypertrophy or histological fibrosis and normal coronary arteries. Dilated, unlike hypertrophic, cardiomyopathy is rarely familial, and the few families previously described have had either sudden death or a benign clinical course as prominent features. ${ }^{1}$ The sporadic form of dilated cardiomyopathy in these brothers was probably inherited.- $M$ V TOBIN, I T GILMORE, et al, Broadgreen Hospital, Liverpool L14 3LB. (Accepted 29 October 1984) 1 Ross RS, Bulkley BH, Hutchins GM, et al. Idiopathic familial myocardiopathy in three genera-
tions: a clinical and pathologic study. Am Hearl $\mathcal{F} 1978 ; 96: 170-9$.

\section{Case clustering in acute epiglottiditis}

Two first cousins were admitted during the same week with acute epiglottiditis, diagnosed on the basis of direct laryngoscopy and blood cultures. They had been in contact with each other three days previously. Two further siblings concurrently suffered a feverish illness and, in view of this apparent case cluster, were treated with ampicillin. Such case clustering is well documented in meningitis due to Haemophilus influenzae $e^{1}$ but has only once been reported between close family members in acute epiglottiditis.- ROWENA $M$ RYAN, ROGER F GRAY, Addenbrooke's Hospital, Cambridge. (Accepted 29 October 1984)

1 Campbell LR, Zedd AJ, Michaels RH. Household spread of infection due to Haemophilus influenzae type B. Pediatrics 1980;66:115-7.

\section{Reduction in numbers of $\mathrm{HBsAg}$ positive blood donors}

Since September 1983 the DHSS leaflet AIDS and how it concerns blood donors has been distributed to every blood donor in the Trent region and made available in departments of genitourinary medicine. Eight donors (three men) positive for hepatitis B surface antigen (HBsAg) were detected in 1983-4 (September to September), whereas 18 (13 men) were detected in 1981-2 and 21 (16 men) in 1982-3. The total number of blood donations rose by $10 \%$ over the three years. We believe that voluntary self exclusion by donors in AIDS high risk groups has led to a reduction in numbers of HBsAg positive donors. - $V$ JAMES, P A NUTTALL, National Blood Transfusion Service, Regional Transfusion Centre, Sheffield S5 7JN. (Accepted 5 November 1984)

\section{Purulent pericarditis: a rare complication of appendicitis}

A previously healthy 17 year old girl presented in cardiac tamponade after three weeks of general malaise and diarrhoea. Examination showed a right iliac fossa mass. Pericardiocentesis produced $900 \mathrm{ml}$ pus. At laparotomy there was pus in the right iliac fossa containing several free faecoliths, although there was no identifiable appendix. Pus from both sites grew an identical Escherichia coli and anaerobes, and, in addition, the pericardial pus grew Streptococcus milleri. She was treated with benzyl penicillin, gentamicin, and metronidazole and made a full recovery. To our knowledge this rare complication of a common condition has not previously been reported. $-M$ O COUPE, J B CHAMBERS, Cardiac Unit, St George's Hospital, London SW17 0QT. (Accepted 13 November 1984)

"Unreviewed Reports" aims at publishing very brief findings quickly, without the usual external peer review. Each item should be no more than 100 words long, with a title of up to 10 words, only one reference, and no more than two named authors (et al is allowed). Authors of papers about side effects must have reported them to the Committee on Safety of Medicines and the manufacturers. Correspondence asking for further details about these items should be sent directly to the authors, who should be willing to supply answers. 